\title{
Deriving object visibilities from interferograms obtained with a fiber stellar interferometer
}

\author{
V. Coudé du Foresto ${ }^{1, \star}$, S. Ridgway ${ }^{2}$, and J.-M. Mariotti ${ }^{3}$ \\ 1 Max-Planck-Institut für Astronomie, Königstuhl 17, 69117 Heidelberg, Germany \\ 2 Kitt Peak National Observatory, National Optical Astronomy Observatories, P.O. Box 26732, Tucson, AZ 85726, U.S.A. ${ }^{\star \star}$ \\ 3 Observatoire de Paris, DESPA, 5 place J. Janssen, 92195 Meudon Cedex, France
}

Received February 12; accepted June 7, 1996

\begin{abstract}
A method is given for extracting object visibilities from data provided by a long baseline interferometer, where the beams are spatially filtered by single-mode fibers and interferograms are obtained as scans around the zero optical pathlength difference. It is shown how the signals can be corrected from the wavefront perturbations caused by atmospheric turbulence. If the piston perturbations are also removed, then the corrected data contain both spatial and spectral information on the source (double Fourier interferometry). When the piston cannot be removed, object phase and spectral information are lost, and the observable (free of detector noise bias) is the squared modulus of the coherence factor, integrated over the optical bandpass. In a fiber interferometer this quantity leads to very accurate object visibility measurements because the transfer function does not involve an atmospheric term. The analysis also holds for a more classical pupil plane interferometer which does not take advantage of the spatial filtering capability of single-mode fibers. In that case however, the transfer function includes a turbulence term that needs to be calibrated by statistical methods.
\end{abstract}

Key words: instrumentation: interferometers methods: data analysis - atmospheric effects - infrared: general - techniques: interferometry

\section{Introduction}

Amplitude interferometry is a unique tool to observe astronomical sources with an angular resolution well beyond

Send offprint requests to: V. Coudé du Foresto

(foresto@hplyot.obspm.fr).

* Visiting astronomer, Kitt Peak National Observatory.

** NOAO is operated by the Association of Universities for Research in Astronomy, Inc., under cooperative agreement with the National Science Foundation. the diffraction limit of monolithic telescopes. The basic Michelson stellar interferometer measures the coherence factor $^{1} \boldsymbol{\mu}$ between two independent pupils, which is linked to the Fourier component of the object intensity distribution (or object visibility $\mathbf{V}$ ) at the spatial frequency corresponding to the baseline formed by the pupils.

One of the main challenges of Michelson stellar interferometry at optical and infrared wavelengths is the calibration of coherence factor measurements. The fringe visibility differs from the object visibility because the instrument and the atmosphere have their own interferometric efficiencies which result in an instrumental transfer function $\mathbf{T}_{\mathrm{i}}$, and an atmospheric transfer function $\mathbf{T}_{\mathrm{a}}$ :

$\boldsymbol{\mu}=\mathbf{T}_{\mathrm{i}} \mathbf{T}_{\mathrm{a}} \mathbf{V}$

Thus an accurate knowledge of both transfer functions is required to obtain a good estimate of $\mathbf{V}$. A well-designed interferometer is usually stable enough so that the calibration of $\mathbf{T}_{\mathrm{i}}$ is not a major issue. But the interferometric efficiency of the atmosphere, which is affected by the loss of coherence caused by phase corrugations on each pupil, depends on the instantaneous state of the turbulent wavefronts. Thus $\mathbf{T}_{\mathrm{a}}$ is a random variable, whose statistics is linked to the evolution of the seeing and is not even stationary. In a classical interferometer there is no way to directly calibrate $\mathbf{T}_{\mathrm{a}}$ (short of sensing at all times the complete shape of the corrugated wavefronts) and the only option is a delicate, statistical calibration on a series of measurements. Then obtaining object visibilities with a relative accuracy of $10 \%$ or better is difficult to achieve; yet such a performance is insufficient for many astrophysical problems.

This difficulty can be overcome by using single-mode fibers to spatially filter the incoming beams. In singlemode fibers the normalized radiation profile is determined

\footnotetext{
1 As far as coherence is concerned, this paper follows the terminology and notations of Goodman (1985). However the term "fringe visibility" will sometimes be used as a synonym for coherence factor.
} 
by the waveguide physical properties (Neumann 1988), not by the input wavefront, and the phase is constant across the guided beam. On the other hand, the intensity of the guided radiation depends on the electromagnetic field amplitude distribution in the focal plane of the telescope and may vary with time if the image is turbulent. Thus single-mode fibers force the transverse coherence of the radiation and transform wavefront phase corrugations into intensity fluctuations of the light coupled into the fibers. Unlike wavefront perturbations however, intensity fluctuations can easily be monitored and used during the data reduction process to correct each interferogram individually against the effects of atmospheric turbulence.

The correction capability was first demonstrated in a fiber unit set up between the two auxiliary telescopes of the McMath-Pierce solar tower on Kitt Peak Observatory, which transformed the telescope pair into a stellar interferometer (Coudé du Foresto et al. 1991). The prototype instrument (named FLUOR for Fiber Linked Unit for Optical Recombination) observed a dozen stars with statistical errors smaller than $1 \%$ on the object visibilities. The same fiber unit is now routinely used as part of the instrumentation in the IOTA (Infrared and Optical Telescope Array) interferometer at the Fred Lawrence Whipple Observatory on Mt Hopkins (Carleton et al. 1994). Some of the results obtained with FLUOR on IOTA can be found in Perrin et al. (1997).

This paper presents the specific data reduction procedure used to extract visibility measurements from the raw interferograms obtained with FLUOR. The procedure can also be applied (with minor modifications that are explained in Sect. 9) even if the interferometer does not involve fiber optics. In that case, however, the spatial filtering advantage is lost.

The organization of the paper is as follows: in Sect. 2 is briefly described the conceptual design of a FLUOR-type interferometer, and the principle of interferogram correction is shown on a simple example.

Before we can derive the full analytical expression of a wide band interferogram (Sect. 5), we need to specify two important preliminary assumptions (Sect. 3) and to understand the photometric behavior of the system (Sect. 4), i.e. the proportionality relationships that link the diverse outputs when light is incoherently recombined. Section 4 is specific to the use of a triple fiber coupler and can be skipped in a first reading. From the expression of a raw interferogram, obtained in Sect. 5, can be derived an expression for the corrected interferogram, which itself leads to an expression for the squared modulus of the wide band fringe visibility (Sect. 6). Real data are affected by noise: estimation strategies and noise sources are discussed in Sect. 7. Finally, some practical considerations are developed in Sect. 8 and a generalization to non-fiber interferometers is proposed in Sect. 9.

Throughout the paper, the data reduction procedure will be illustrated with examples from actual data. They were obtained on $\alpha$ Boo (Arcturus) with the original FLUOR unit set up between the two $0.8 \mathrm{~m}$ telescopes (separated by $5.5 \mathrm{~m}$ ) of the McMath-Pierce tower (Coudé du Foresto et al. 1991). The unit included fluoride glass fibers and couplers, four InSb photometers, and was operated in the infrared $K$ band $(2 \mu \mathrm{m} \leq \lambda \leq 2.4 \mu \mathrm{m})$. The telescopes had entirely passive optics, without even active guiding (tip-tilt correction). The sample data is a batch of 122 interferograms recorded on 7 April 1992 between $7 \mathrm{~h} 19$ and 8h04 UT, in mediocre seeing conditions (more than 1.5 arcsec).

\section{Principles of a fiber interferometer}

It is beyond the scope of this paper to describe the details of a fiber interferometer. This has been done elsewhere (Coudé du Foresto 1994). What is shown here is only a conceptual description of a FLUOR-type instrument (Fig. 1) and the principles of operation.

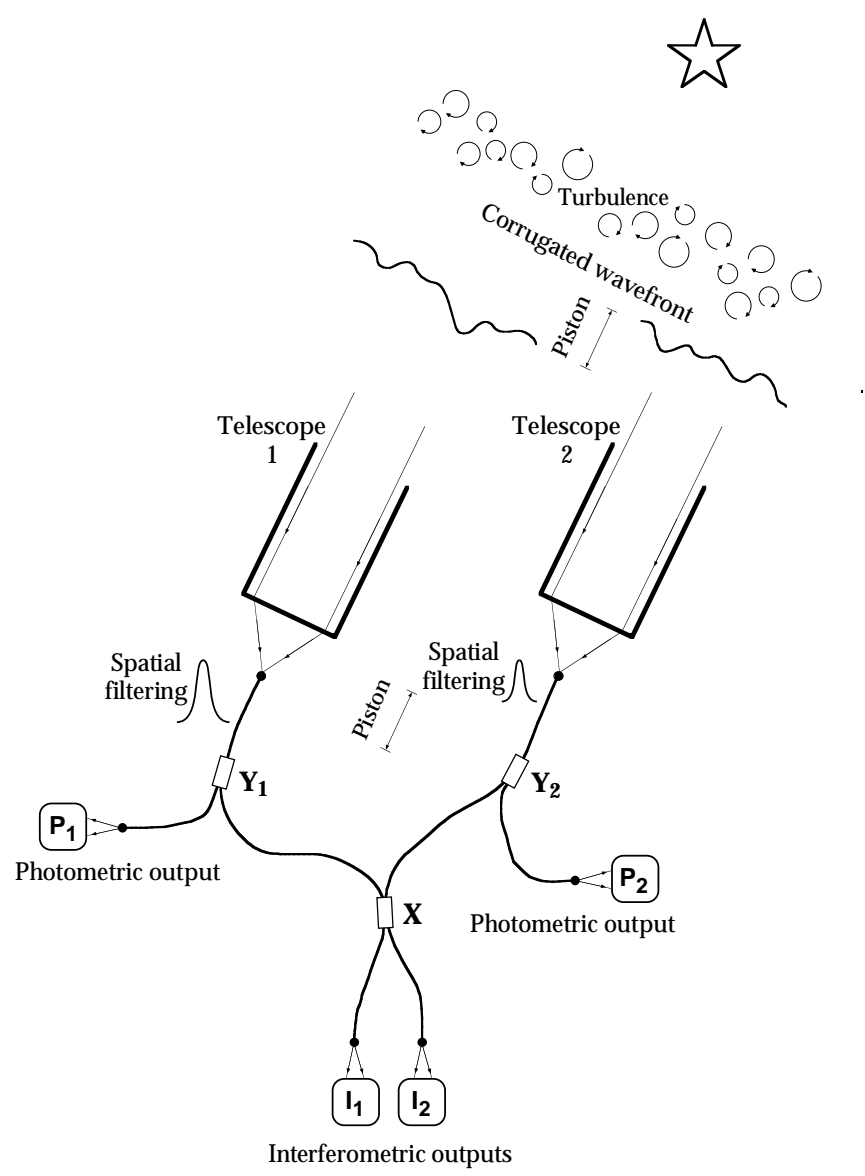

Fig. 1. Conceptual design of a stellar fiber interferometer

Two different pupils independently collect the radiation from an astronomical source, and each telescope focuses the light onto the input head of a single-mode optical fiber. 
The observed object is considered as unresolved by a single pupil and its spectral intensity distribution at the focus of the telescopes is $B_{0} B(\sigma)$, with $B(\sigma)$ normalized so that

$\int_{0}^{+\infty} B(\sigma) \mathrm{d} \sigma=1$.

Starlight injection into the waveguide occurs as the focal electric field $\mathbf{E}_{\text {focus }}$ excites the fundamental mode of the fiber $\mathbf{E}_{\text {fiber. }}$. The instantaneous coupling efficiency $\rho$ is determined by the overlap integral between the distribution of the electric fields in the focal plane and in the guided mode (Shaklan \& Roddier 1988):

$\rho=\frac{\left|\int_{A_{\infty}} \mathbf{E}_{\text {focus }} \mathbf{E}_{\text {fiber }}^{*} \mathrm{~d} A\right|^{2}}{\int_{A_{\infty}}\left|\mathbf{E}_{\text {focus }}\right|^{2} \mathrm{~d} A \int_{A_{\infty}}\left|\mathbf{E}_{\text {fiber }}\right|^{2} \mathrm{~d} A}$,

where the integration domain extends at infinity in a transverse plane and the symbol * denotes a complex conjugate.

The radiation is then guided by the fiber down to the recombination point, where correlation between the two beams occurs in a single-mode directional coupler $(X)$. The two complementary outputs of the coupler are measured by photometers which produce the interferometric signals $I_{1}$ and $I_{2}$. Two auxiliary couplers $Y_{1}$ and $Y_{2}$ derive part of the light at each telescope so that the coupling fluctuations can be monitored by the photometers which produce the photometric signals $P_{1}$ and $P_{2}$.

With a delay line, the observer has the capacity to control the overall optical pathlength difference (OPD) from the source to the recombination point. During data acquisition, the OPD is scanned around the zero pathlength difference. The nominal scanning speed $v$ is the algebraic sum of the internal OPD modulation introduced by the delay line and the external OPD modulation due to diurnal motion.

Thus a complete data set for a single interferogram contains the collection of four signals $I_{1}, I_{2}, P_{1}$ and $P_{2}$, sampled and digitized during a scan. It also includes the background current sequences for each photometer (the sum of the dark current and the background signal), which are preferably acquired just after each scan. It is assumed in what follows that the electrical offsets have been adjusted in such a way that the average value of all background currents is zero. To reduce statistical errors on the results, a batch of a few tens to a few hundred interferograms is recorded for a given source and instrumental configuration.

A simple example, using a monochromatic source at wave number $\sigma$, will help us understand how fiber interferograms can be corrected from the turbulence induced coupling fluctuations. Neglecting transmission and proportionality factors that are detailed in Sect. 4 , the expression of a generic interferogram $I$ is

$I(x)=P_{1}(x)+P_{2}(x)+2 \sqrt{P_{1}(x) P_{2}(x)} \mu \cos (2 \pi \sigma x+\Phi),(4)$ where $\mu$ is the modulus of the complex coherence factor, $x$ the optical path difference, and $\Phi$ a phase term. From this and with the knowledge of $P_{1}$ and $P_{2}$, it is easy to build the corrected interferogram whose modulated part is:

$$
\begin{aligned}
I_{\mathrm{cor}}(x) & =\frac{I(x)-P_{1}(x)-P_{2}(x)}{2 \sqrt{P_{1}(x) P_{2}(x)}} \\
& =\mu \cos (2 \pi \sigma x+\Phi) .
\end{aligned}
$$

The quantity $1+I_{\text {cor }}$ is the normalized interferogram that would have been observed if there had been no atmospheric turbulence, i.e. if $P_{1}$ and $P_{2}$ had been equal and constant.

In Sect. 5 is established a more rigorous expression of the interferogram for a monochromatic and for a wide band source.

\section{Preliminary assumptions}

Two important assumptions have to be made before we go any further. Those conditions are not necessarily fully satisfied, but they are required if one wants to develop an analytical expression of the interferogram.

\subsection{Absence of differential piston}

In a modal description of atmospheric turbulence, the piston corresponds to the most fundamental perturbation, i.e. the fluctuations of the average phase of the corrugated wavefront. The piston mode on a single pupil does not modify the state of coherence of that pupil and therefore, does affect neither the image quality nor the coupling efficiency into a single-mode fiber. The differential piston between two independent pupils, however, is equivalent to the addition of a small random delay in the OPD.

Recently, Perrin (1997) proposed a numerical method to remove the differential piston after data acquisition. The piston can also be eliminated in the instrument before data acquisition if the pupils are cophased with a fringe tracker, as it is the case for example in the Mark III interferometer (Shao et al. 1988). A fringe tracker based on guided optics has already been proposed (Rohloff \& Leinert 1991). Cophasing the pupils offers the additional advantage to considerably improve the sensitivity because integration times can in principle be arbitrarily long (Mariotti 1993), but it requires to build a dedicated active system.

Without piston, the OPD variation is uniform. The signals are recorded as time sequences, but if we assume that the fringe speed $v$ is constant during a scan there is a direct linear relationship between the time variable $t$ and the position variable (the global OPD) $x$. The signals are sampled at equal time intervals $\delta t$, which correspond to equal length intervals $\delta x=v \delta t$.

When taking the Fourier transform of the signals, the conjugate variable with respect to position $x$ is the wave 
number $\sigma$, and the frequency $f=v \sigma$ is the conjugate variable for the temporal sequences. It is important to keep this duality in mind in order to be able to reason alternatively in terms of time/frequency or position/wave number. Actually, in this paper either one or the other variable pair is used depending on the needs, and the variable change that it sometimes implies shall be implicit.

\subsection{Chromaticism of the starlight injection}

Usually the starlight injection efficiency $\rho$ is both a function of time $t$ and of wave number $\sigma$, since the structures of both $\mathbf{E}_{\text {fiber }}$ and $\mathbf{E}_{\text {focus }}$ depend on wavelength, and $\mathbf{E}_{\text {focus }}$ is determined by the instantaneous state of the atmospheric turbulence. For what follows it is necessary to assume that the time and wave number variables can be separated in the coupling efficiency coefficient, so that we can write

$\rho(t, \sigma)=\rho_{t}(t) \rho_{\sigma}(\sigma)$.

A heuristic justification is given here. For a diffraction limited image, the electric field morphology at the focus of the telescope depends on wavelength $\lambda$ only through a scaling factor. Within the practical range of optical frequencies at which a single-mode fiber can be operated, the fundamental mode can be approximated by a Gaussian function whose width is proportional to $\lambda$ (Neumann 1988). Thus the two fields change homothetically with respect to wavelength, and the overlap integral (Eq. 3) remains almost unchanged. It follows that the injection efficiency is quasi achromatic for a diffraction limited image.

Things are different for a stellar source, but if we assume that the turbulence is weak $\left(d / r_{0} \leq 4\right.$, where $d$ is the diameter of the pupil and $r_{0}$ the Fried parameter (Fried 1966)), tip-tilt modes dominate the atmospheric turbulence (Noll 1976) and the image of the star can be modeled by a unique speckle randomly walking around its nominal position. The speckle offset with respect to the fiber core is a function of time exclusively, whereas the sensitivity of $\rho$ to that offset depends on the color only. It is thus reasonable to assume that the time and wave number variables can be separated.

\section{Photometric properties of the system}

The aim of this section is to determine, when photometric intensities $P_{1}$ and $P_{2}$ are recorded at the outputs of the $Y$ couplers, what are the actual intensities of the beams that are being correlated in the $X$ coupler. We need to establish what proportionality factors link the outputs of the interferometric $X$ coupler and the outputs of the photometric $Y$ couplers. So for this section it is assumed that the recombination is fully incoherent.

\subsection{Monochromatic signals}

The monochromatic signal produced at any given time by the photometric detector $P_{j}(j=1$ or 2$)$ is proportional to its overall gain $g_{P j}$ and to the transmission $t_{P j}$ of the coupler $Y_{j}$ towards the output $P_{j}$ :

$P_{\sigma, j}=g_{P j}(\sigma)\left[t_{P j}(\sigma) B_{0} B(\sigma) \rho_{t, j} \rho_{\sigma, j}(\sigma)\right]$.

For what follows it is convenient to introduce the global efficiency $\eta_{P j}$ of the photometric channel $P_{j}$ :

$\eta_{P j}(\sigma)=g_{P j} t_{P j} \rho_{\sigma, j}$,

which leads to

$P_{\sigma, j}=B_{0} B(\sigma) \eta_{P j}(\sigma) \rho_{t, j}$.

The signal provided by the interferometric detectors $I_{1}$ and $I_{2}$ is proportional to the optical powers $E_{1}^{2}$ and $E_{2}^{2}$ at the output of the $X$ coupler, and to the gain of the photometers:

$I_{\sigma, 1}=g_{\mathrm{I} 1}(\sigma) E_{1}^{2}$
$I_{\sigma, 2}=g_{\mathrm{I} 2}(\sigma) E_{2}^{2}$.

Optical powers at the inputs and outputs of $X$ are linked by a transmission matrix:

$$
\left(\begin{array}{l}
E_{1}^{2} \\
E_{2}^{2}
\end{array}\right)=\left(\begin{array}{ll}
t_{X 11} & t_{X 12} \\
t_{X 21} & t_{X 22}
\end{array}\right)\left(\begin{array}{l}
t_{Y 1} B_{0} B(\sigma) \rho_{t, 1} \rho_{\sigma, 1}(\sigma) \\
t_{Y 2} B_{0} B(\sigma) \rho_{t, 2} \rho_{\sigma, 2}(\sigma)
\end{array}\right),
$$

where $t_{Y j}$ is the transmission of the coupler $Y_{j}$ towards the $X$ coupler, and $t_{X i j}$ is the transmission of the $X$ coupler from input (telescope) $j$ towards output $i$.

Here it should be noted that, since most fiber couplers are chromatic (and because of aging processes, their chromaticism can evolve over the years), the coefficients $t_{X i j}$ and $t_{Y j}$ are $\sigma$-dependent and cannot be known with a sufficient accuracy. There is also no way to access the raw coupling efficiencies. Thus the only matrix that can actually be measured links the photometric signals to the interferometric signals:

$$
\left(\begin{array}{l}
I_{\sigma, 1} \\
I_{\sigma, 2}
\end{array}\right)=\left(\begin{array}{ll}
\kappa_{11}(\sigma) & \kappa_{12}(\sigma) \\
\kappa_{21}(\sigma) & \kappa_{22}(\sigma)
\end{array}\right)\left(\begin{array}{l}
P_{\sigma, 1} \\
P_{\sigma, 2}
\end{array}\right)
$$

Combining the relations 7, 11 and 12 leads to

$\kappa_{i j}(\sigma)=\frac{g_{I i}}{g_{P j}} \frac{t_{X i j} t_{Y j}}{t_{P j}}$.

\subsection{Wide band signals}

The wide band photometric relationships are obtained by integration over $\sigma$. For the $P_{j}$ detectors:

$$
\begin{aligned}
P_{j} & =\int_{0}^{+\infty} P_{\sigma, j}(\sigma) \mathrm{d} \sigma \\
& =B_{0} \rho_{t, j} \int_{0}^{+\infty} B(\sigma) \eta_{P j}(\sigma) \mathrm{d} \sigma .
\end{aligned}
$$


From this follows:

$B_{0} \rho_{t, j}=\frac{P_{j}}{\bar{\eta}_{P j}}$,

where

$\bar{\eta}_{P j}=\int_{0}^{+\infty} B(\sigma) \eta_{P j}(\sigma) \mathrm{d} \sigma$

is the global wide band efficiency of the photometric channel $P_{j}$.

According to Eq. (12), the monochromatic signal $I_{\sigma, i}$ provided by detector $I_{i}$ is linked to $P_{\sigma, 1}$ and $P_{\sigma, 2}$ by

$$
\begin{aligned}
I_{\sigma, i}(\sigma)= & \kappa_{i 1}(\sigma) P_{\sigma, 1}+\kappa_{i 2}(\sigma) P_{\sigma, 2} \\
= & \kappa_{i 1}(\sigma) B_{0} B(\sigma) \eta_{P 1}(\sigma) \rho_{t, 1} \\
& +\kappa_{i 2}(\sigma) B_{0} B(\sigma) \eta_{P 2}(\sigma) \rho_{t, 2}
\end{aligned}
$$

and an integration yields

$$
\begin{aligned}
I_{i} & =\sum_{j=1,2} B_{0} \rho_{t, j} \int_{0}^{+\infty} \kappa_{i j}(\sigma) \eta_{P j}(\sigma) B(\sigma) \mathrm{d} \sigma \\
& =\sum_{j=1,2} \frac{P_{j}}{\bar{\eta}_{P j}} \int_{0}^{+\infty} \kappa_{i j}(\sigma) \eta_{P j}(\sigma) B(\sigma) \mathrm{d} \sigma .
\end{aligned}
$$

Thus a linear relationship links the two wide band signal pairs:

$$
\left(\begin{array}{l}
I_{1} \\
I_{2}
\end{array}\right)=\left(\begin{array}{ll}
\bar{\kappa}_{11} & \bar{\kappa}_{12} \\
\bar{\kappa}_{21} & \bar{\kappa}_{22}
\end{array}\right)\left(\begin{array}{l}
P_{1} \\
P_{2}
\end{array}\right)
$$

with:

$$
\bar{\kappa}_{i j}=\frac{1}{\bar{\eta}_{P j}} \int_{0}^{+\infty} \kappa_{i j}(\sigma) \eta_{P j}(\sigma) B(\sigma) \mathrm{d} \sigma .
$$

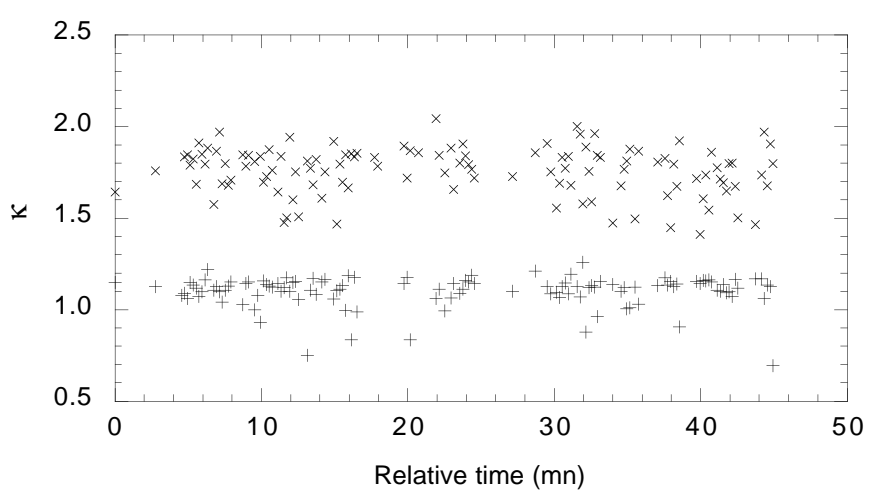

Fig. 2. The $\bar{\kappa}_{11}(+)$ and $\bar{\kappa}_{12}(\times)$ coefficients, as measured on $\alpha$ Boo (Arcturus) in a batch of 122 scans

The main interest of the $\left(\bar{\kappa}_{i j}\right)$ transfer matrix is that it can be evaluated directly from the data, without requiring an a priori knowledge of the individual transmissions and gains in the system. The evaluation is performed by adjusting a least square fit of a linear combination of $P_{1}$ and $P_{2}$ to $I_{1}$ for the $\bar{\kappa}_{1 j}$, and to $I_{2}$ for the $\bar{\kappa}_{2 j}$. Figure 2 shows an example of a series of measurements of $\bar{\kappa}_{11}$ and $\bar{\kappa}_{12}$. In order to reduce statistical errors, for each $\bar{\kappa}_{i j}$ all measurements in a batch are averaged to produce the value adopted for the rest of the data reduction procedure.

\section{Coherent recombination}

For data reduction both interferometric signals are processed independently. We shall consider only one of them, which will help us simplify the notations as we can then drop index $i$. Thus for example, when the recombination is incoherent the wide band interferometric signal can be expressed as

$I=\bar{\kappa}_{1} P_{1}+\bar{\kappa}_{2} P_{2}$,

where the $\bar{\kappa}_{j}$ are the proportionality factors detailed in Sect. 4.

To establish an expression of the intensity after coherent recombination in the $X$ coupler, we first describe the monochromatic interferogram at wave number $\sigma$; the wide band interferogram will then be obtained by integration over the optical bandpass of the system.

\subsection{Monochromatic interferogram}

At the recombination point in the correlator the complex representations of the electric fields of the two guided beams have the general expression:

$\mathbf{E}_{1}(t)=E_{1} \mathrm{e}^{j\left(\phi_{1}-\omega t\right)}$

and

$\mathbf{E}_{2}(t)=E_{2} \mathrm{e}^{j\left(\phi_{2}-\omega t\right)}$

where $\omega=2 \pi c \sigma$ is the angular pulsation and $\phi_{i}$ the phase accumulated from the source to the recombination point through channel $i$. The correlator sums the instantaneous amplitudes of the electric fields and the instantaneous amplitude at its output is:

$$
\begin{aligned}
\mathbf{E}(t) & =\mathbf{E}_{1}(t)+\mathbf{E}_{2}(t) \\
& =E_{1} \mathrm{e}^{j\left(\phi_{1}-\omega t\right)}+E_{2} \mathrm{e}^{j\left(\phi_{2}-\omega t\right)} .
\end{aligned}
$$

What is measured by the observer is a quantity proportional to the average (over a period much greater than the coherence time of the radiation) of the squared modulus of the amplitude:

$$
\begin{aligned}
I_{\sigma} & =g_{I}\left[\left\langle\mathbf{E}(t) \mathbf{E}^{*}(t)\right\rangle\right] \\
& =g_{I}\left[E_{1}^{2}+E_{2}^{2}+2\left\langle\mathbf{E}_{1}(t) \mathbf{E}_{2}^{*}(t)\right\rangle\right] \\
& =g_{I}\left[E_{1}^{2}+E_{2}^{2}+2 E_{1} E_{2} \operatorname{Re}\left\{\gamma_{12}\right\}\right] .
\end{aligned}
$$

The quantity $\gamma_{12}$ is the complex degree of coherence (Goodman 1985) between the signals collected by each 
fiber. Here because the source is by definition monochromatic (with an optical frequency $\nu$ and a wave number $\sigma$ ), the complex degree of coherence takes the simplified form of an oscillating function of the delay $\tau$ between the two waves:

$$
\begin{aligned}
\gamma_{12} & =\boldsymbol{\mu}_{12} \mathrm{e}^{-j 2 \pi \nu \tau} \\
& =\boldsymbol{\mu}_{12} \mathrm{e}^{-j 2 \pi \sigma x},
\end{aligned}
$$

where $\boldsymbol{\mu}_{12}=\mu_{12} \mathrm{e}^{j \Phi_{12}}$ is the complex coherence factor between the two beams at the recombination point. It is the product of the complex object visibility $\mathbf{V}_{12}^{(\star)}=V_{12}^{(\star)} \mathrm{e}^{j \Phi_{12}^{(\star)}}$ at the input (the entrance pupils) of the interferometer by an instrumental transfer function $\mathbf{T}_{\mathrm{i}}=T_{\mathrm{i}} \mathrm{e}^{j \Phi_{\mathrm{i}}}$ :

$\boldsymbol{\mu}_{12}=\mathbf{T}_{\mathrm{i}} \mathbf{V}_{12}^{(\star)}$.

According to the Van Cittert-Zernike theorem (Born \& Wolf 1980; Goodman 1985), the quantity $\mathbf{V}_{12}^{(\star)}$ is also the monochromatic complex visibility of the object at the spatial frequency corresponding to the interferometric baseline vector. The modulation transfer function $T_{\mathrm{i}}$ of the instrument expresses mainly the frequency response of the detectors and a coherence loss due to polarization mismatch in both radiations. The phase term $\Phi_{\mathrm{i}}$ includes dispersion in the guided optics components (Coudé du Foresto et al. 1995), and possible differential phase jumps when reflecting on telescope mirrors.

It should be emphasized that the transfer function, as defined here, is purely instrumental and does not involve atmospheric turbulence. Assuming that the instrument is stable enough, the impulse response of the system can be calibrated on a reference object whose complex visibility $\mathbf{V}_{12}^{(\mathrm{ref})}$ is well known (it can be for example an unresolved point source for which $\mathbf{V}_{12}^{(\mathrm{ref})}=1$ ). If $\boldsymbol{\mu}_{12}^{(\mathrm{ref})}$ is the complex coherence factor measured on the reference, the instrumental transfer function is

$\mathbf{T}_{\mathrm{i}}=\frac{\boldsymbol{\mu}_{12}^{(\mathrm{ref})}}{\mathbf{V}_{12}^{(\mathrm{ref})}}$.

The modulus and phase of the Fourier transform of the object are then given by

$V_{12}^{(\star)}=\mu_{12} / T_{\mathrm{i}}$

$\Phi_{12}^{(\star)}=\Phi_{12}-\Phi_{\mathrm{i}}$.

Let us now return to the general expression of the interferogram (Eq. 25), which can be rewritten, with relation 26 in mind,

$$
\begin{aligned}
I_{\sigma}= & g_{I} E_{1}^{2}+g_{I} E_{2}^{2} \\
& +2 g_{I} E_{1} E_{2} \mu_{12}(\sigma) \cos \left(2 \pi \sigma x+\Phi_{12}(\sigma)\right) .
\end{aligned}
$$

Thanks to the results of the previous section (Eqs. 9, 12 and 15), we can relate the squared monochromatic amplitudes of the fields, which cannot be accessed directly, to the photometric signals $P_{1}$ and $P_{2}$ which are measurable quantities:

$$
\begin{aligned}
g_{I} E_{j}^{2} & =\kappa_{j}(\sigma) P_{\sigma, j} \\
& =\kappa_{j}(\sigma) B_{0} B(\sigma) \eta_{P j}(\sigma) \rho_{t} \\
& =\kappa_{j}(\sigma) \frac{P_{j}}{\bar{\eta}_{P j}} B(\sigma) \eta_{P j}(\sigma) .
\end{aligned}
$$

Defining $P=\sqrt{P_{1} P_{2}}$ and $\kappa=\sqrt{\kappa_{1} \kappa_{2}}$, we have for the cross term:

$$
\begin{aligned}
g_{I} E_{1} E_{2} & =P B(\sigma) \kappa(\sigma) \frac{\sqrt{\eta_{P 1}(\sigma) \eta_{P 2}(\sigma)}}{\sqrt{\bar{\eta}_{P 1} \bar{\eta}_{P 2}}} \\
& =P B(\sigma) \kappa^{\prime}(\sigma) .
\end{aligned}
$$

Since the photometric signals $P_{1}$ and $P_{2}$ vary with time (i.e., with the OPD $x$ ), the monochromatic interferogram can be written as:

$$
\begin{aligned}
I_{\sigma}(x)= & \kappa_{1}(\sigma) \frac{P_{1}(x)}{\bar{\eta}_{P 1}} B(\sigma) \eta_{P 1}(\sigma) \\
& +\kappa_{2}(\sigma) \frac{P_{2}(x)}{\bar{\eta}_{P 2}} B(\sigma) \eta_{P 2}(\sigma) \\
& +2 P(x) B(\sigma) \kappa^{\prime}(\sigma) \mu_{12}(\sigma) \cos \left(2 \pi \sigma x+\Phi_{12}(\sigma)\right) .
\end{aligned}
$$

It is the sum of the scaled photometric signals (an additive scintillation noise) and a sinusoid whose amplitude is modulated by the geometric average of the injection efficiencies (a multiplicative noise).

\subsection{Wide band interferogram}

The wide band interferogram is calculated by integration of the monochromatic signals over the optical bandpass:

$I(x)=\int_{0}^{+\infty} I_{\sigma}(x) \mathrm{d} \sigma$.

After having extended to negative wave numbers the validity range of all functions of $\sigma$ (their value is set to 0 for $\sigma<0$ ), Eq. (34) yields:

$$
\begin{aligned}
I(x)= & P_{1}(x) \frac{1}{\bar{\eta}_{P 1}} \int_{0}^{+\infty} \kappa_{1}(\sigma) \eta_{P 1}(\sigma) B(\sigma) \mathrm{d} \sigma \\
& +P_{2}(x) \frac{1}{\bar{\eta}_{P 2}} \int_{0}^{+\infty} \kappa_{2}(\sigma) \eta_{P 2}(\sigma) B(\sigma) \mathrm{d} \sigma \\
& +\int_{-\infty}^{+\infty} P(x) B(\sigma) \kappa^{\prime}(\sigma) \mu_{12}(\sigma) \mathrm{e}^{j \Phi_{12}(\sigma)} \mathrm{e}^{j 2 \pi \sigma x} \mathrm{~d} \sigma \\
& +\int_{-\infty}^{+\infty} P(x) B(\sigma) \kappa^{\prime}(\sigma) \mu_{12}(\sigma) \mathrm{e}^{-j \Phi_{12}(\sigma)} \\
& \mathrm{e}^{-j 2 \pi \sigma x} \mathrm{~d} \sigma .
\end{aligned}
$$

The first two lines of Eq. (35) are related to the wide band scintillation noise. The last two lines can be identified with an inverse Fourier transform, and the multiplicative noise 
of the fringe signal in the OPD space becomes a convolution in the wave number space:

$$
\begin{aligned}
& I(x)=\bar{\kappa}_{1} P_{1}(x)+\bar{\kappa}_{2} P_{2}(x) \\
& +\mathcal{F}^{-1}\left\{\tilde{\mathbf{P}}(\sigma) *\left(B(\sigma) \kappa^{\prime}(\sigma) \mu_{12}(\sigma) \mathrm{e}^{j \Phi_{12}(\sigma)}\right)\right\} \\
& +\mathcal{F}^{-1}\left\{\tilde{\mathbf{P}}(\sigma) *\left(B(-\sigma) \kappa^{\prime}(-\sigma) \mu_{12}(-\sigma) \mathrm{e}^{-j \Phi_{12}(-\sigma)}\right)\right\} .
\end{aligned}
$$

In what follows we will use either the symbol $\mathcal{F}$ or the tilde ${ }^{\sim}$ for the Fourier transform (FT) operation. The FT of the interferometric signal is:

$$
\begin{aligned}
\tilde{\mathbf{I}}(\sigma)= & \bar{\kappa}_{1} \tilde{\mathbf{P}}_{1}(\sigma)+\bar{\kappa}_{2} \tilde{\mathbf{P}}_{2}(\sigma) \\
& +\tilde{\mathbf{P}}(\sigma) *\left(B(\sigma) \kappa^{\prime}(\sigma) \mu_{12}(\sigma) \mathrm{e}^{j \Phi_{12}(\sigma)}\right) \\
& +\tilde{\mathbf{P}}(\sigma) *\left(B(-\sigma) \kappa^{\prime}(-\sigma) \mu_{12}(-\sigma) \mathrm{e}^{-j \Phi_{12}(-\sigma)}\right) \\
= & \tilde{\mathbf{I}}_{\mathrm{LF}}+\tilde{\mathbf{I}}_{\mathrm{HF}^{+}}+\tilde{\mathbf{I}}_{\mathrm{HF}^{-}} .
\end{aligned}
$$

If the OPD scanning speed is fast enough for the fringe frequency to be larger than the bandwidth of the coupling fluctuations (typically a few tens of $\mathrm{Hz}$ ), then the interferogram has two distinct components in the frequency space:

- A low frequency scintillation noise, which is the spectrum of the coupling fluctuations into the fibers;

- An interferometric signal at a higher frequency (and its Hermitic counterpart), which is the spectral intensity of the source multiplied by the coherence factor between the two beams and convolved with the FT of the coupling fluctuations.

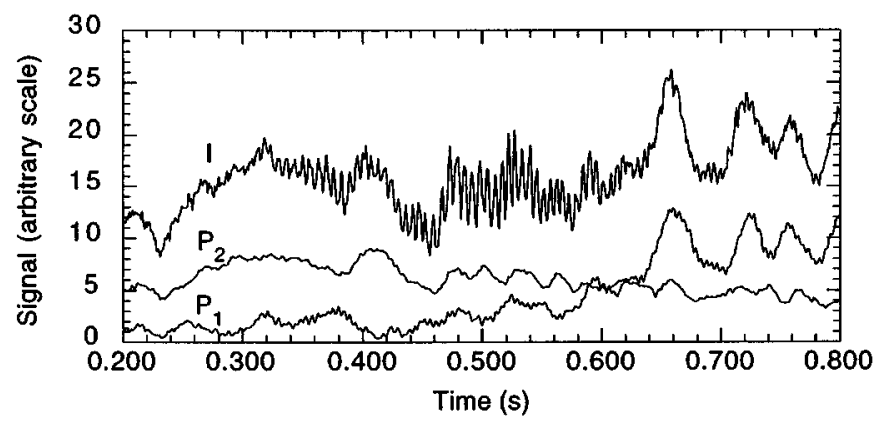

Fig. 3. An example of a time sequence, with the interferometric signal $I(t)$ and the two photometric signals $P_{1}(t)$ and $P_{2}(t)$. Scanning speed is $359 \mu \mathrm{m} / \mathrm{s}$. The duration of the recorded scan is $1.024 \mathrm{~s}$ but only the central $0.6 \mathrm{~s}$ is displayed here to better show the fringes

An example of interferogram obtained on Arcturus is shown Fig. 3, while its one-sided amplitude spectrum is in Fig. 4. During the scan 1024 points have been recorded at a sampling rate of $1 \mathrm{kHz}$. In the time sequence, the number of fringes is substantially larger than what might be expected from an interferogram obtained with a $K$ band

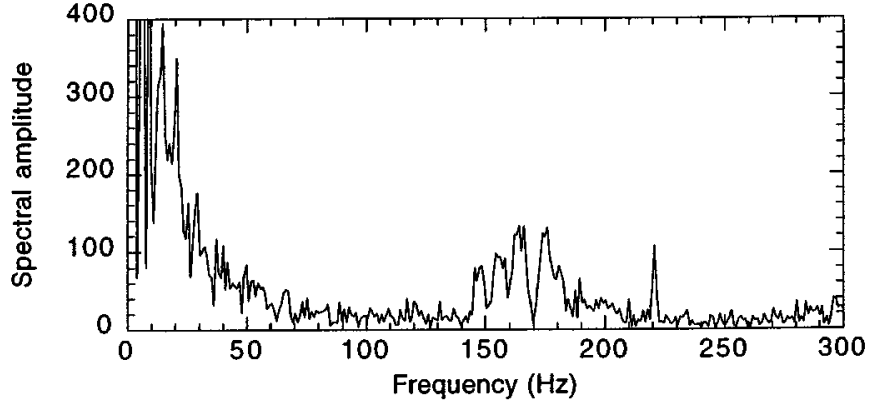

Fig. 4. Modulus of the Fourier Transform of $I(t)$, showing the low frequency scintillation noise and the interferometric signal at a higher frequency

filter. This is caused by chromatic differential dispersion in the waveguides, which affects the phase part $\Phi_{\mathrm{i}}$ of the instrumental transfer function, but not its amplitude $T_{\mathrm{i}}$, and therefore does not have consequences on visibility measurements (Coudé du Foresto et al. 1995). The amplitude spectrum shows the contribution of scintillation noise at low frequencies $(\leq 80 \mathrm{~Hz})$ and the fringe signal at higher frequencies. The spectral width at half maximum of the scintillation noise being only a few Hertz, the convolution broadening of the fringe signal is very small. What is better seen here in the fringe signal is the effect of differential piston, which corrupts the Fourier relationship between the fringe pattern in the OPD (time) domain and the spectral intensity distribution of the source. Here the nominal fringe speed is $v=359 \mu \mathrm{m} / \mathrm{s}$, which corresponds to fringe frequencies ranging from $150 \mathrm{~Hz}$ (for $\lambda=2.4 \mu \mathrm{m}$ ) to $180 \mathrm{~Hz}$ (for $\lambda=2.0 \mu \mathrm{m}$ ). However, because of piston phase perturbations the fringe signal is spread substantially beyond this nominal frequency range, and presents deep spectral features that cannot be attributed to the real spectroscopic lines of Arcturus, a standard K2III star.

\section{Deriving the fringe visibility from an interferogram}

The expression 37 is the basis on which will be derived the coherence factor between the two beams. In a first step we need to correct the interferogram from the turbulence induced modulation of the fringe pattern; then we can derive an expression of the coherence factor that does not depend on turbulence.

\subsection{Correcting the interferogram}

The first operation consists in separating the low- and high frequency components of the signal, to isolate the interferometric signal from the scintillation noise. This is best achieved by a subtraction in the OPD space:

$I_{\mathrm{HF}}(x)=I(x)-\bar{\kappa}_{1} P_{1}(x)-\bar{\kappa}_{2} P_{2}(x)$. 
It is known from Eq. (37) that in the wave number space $\tilde{\mathbf{I}}_{\mathrm{HF}}$ is written as:

$$
\begin{aligned}
\tilde{\mathbf{I}}_{\mathrm{HF}}(\sigma)= & \tilde{\mathbf{P}}(\sigma) *\left[B(\sigma) \kappa^{\prime}(\sigma) \mu_{12}(\sigma) \mathrm{e}^{j \Phi_{12}(\sigma)}\right. \\
& \left.+B(-\sigma) \kappa^{\prime}(-\sigma) \mu_{12}(-\sigma) \mathrm{e}^{-j \Phi_{12}(-\sigma)}\right]
\end{aligned}
$$

which yields by inverse Fourier transform in the OPD domain, where the convolution by the scintillation noise becomes a product:

$$
\begin{aligned}
I_{\mathrm{HF}}(x)= & P(x) \mathcal{F}^{-1}\left\{B(\sigma) \kappa^{\prime}(\sigma) \mu_{12}(\sigma) \mathrm{e}^{j \Phi_{12}(\sigma)}\right. \\
& \left.+B(-\sigma) \kappa^{\prime}(-\sigma) \mu_{12}(-\sigma) \mathrm{e}^{-j \Phi_{12}(-\sigma)}\right\}
\end{aligned}
$$

Dividing by $P(x)$ and going back to the wave number space help us establish an interferometric signal $\tilde{\mathbf{I}}_{\text {cor }}$ which is corrected from the atmosphere induced fluctuations:

$$
\begin{aligned}
\tilde{\mathbf{I}}_{\mathrm{cor}}(\sigma)= & \frac{1}{2} \mathcal{F}\left\{\frac{I_{\mathrm{HF}}(x)}{P(x)}\right\} \\
= & \frac{1}{2} B(\sigma) \kappa^{\prime}(\sigma) \mu_{12}(\sigma) \mathrm{e}^{j \Phi_{12}(\sigma)} \\
& +\frac{1}{2} B(-\sigma) \kappa^{\prime}(-\sigma) \mu_{12}(-\sigma) \mathrm{e}^{-j \Phi_{12}(-\sigma)} .
\end{aligned}
$$

The factor $1 / 2$ was introduced to normalize to 1 the integral of the modulus $\tilde{I}_{\text {cor }}$ of $\tilde{\mathbf{I}}_{\text {cor }}$ in the canonical case where $\kappa^{\prime}=1$ and $\mu_{12}=1$.

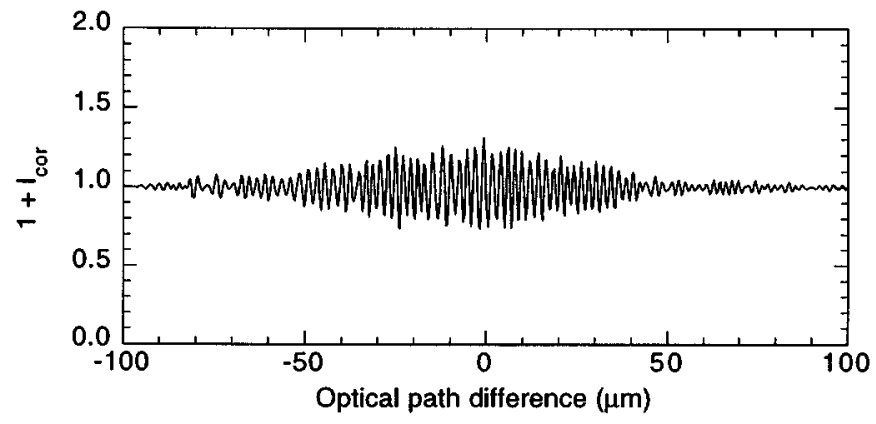

Fig. 5. The interferogram of Fig. 3, after correction

Figure 5 shows the corrected sequence $1+I_{\text {cor }}(x)$ corresponding to the interferogram of Fig. 3.

\subsection{Double Fourier mode}

From the relationship (41), and after calibration of the instrumental transfer function, can be measured the product $\mathbf{V}_{12}^{(\star)}(\sigma) B(\sigma)$ of the object complex visibility by its spectral intensity. To obtain this result, which is at the basis of double Fourier interferometry (Itoh \& Ohtsuka 1986; Mariotti \& Ridgway 1988), three conditions need to be fulfilled:
1. The instrumental response needs to be perfectly known. We have seen that the complex transfer function $\mathbf{T}_{\mathbf{i}}(\sigma)$ could be measured on a reference star; the coefficient $\kappa^{\prime}(\sigma)$ needs also to be calibrated (on a reference star or an internal source);

2. The differential piston between the two pupils must be removed;

3. The decoupling of time and wavelength in the injection efficiency must be effective.

Because it combines spectral information with high angular resolution, the scientific potential of double Fourier interferometry is considerable as it fully exploits the fundamentally tridimensionnal nature of interferometric data. While the spatial coherence of the source (the 2D FT of the object intensity distribution) is explored by the double pupil of the interferometer, its temporal coherence (the 1D FT of its spectral distribution) is explored by the scan around the zero OPD. One can also use the generalized version of the Van Cittert-Zernike theorem (Born \& Wolf 1980), and say that the mutual coherence of the collected beams is linked by a tridimensionnal FT to the spatio-spectral intensity distribution of the object. In the type of interferometer we consider here, each interferogram can in principle lead to the measurement of one line in the interferometric data cube. Although interferometric data cubes are familiar to radio astronomers (Perley 1985), dealing with them in the context of optical and infrared observations is quite new: one can consult Claret et al. (1991) for a first approach.

\subsection{The wide band coherence factor}

In all data obtained so far, there is no piston compensation and the phase information in the interferogram is lost. Acknowledging this situation, we shall concentrate on the squared modulus of the Fourier transform, which is the spectral power density of the corrected interferometric signal:

$$
\begin{aligned}
\tilde{I}_{\mathrm{cor}}^{2}(\sigma)= & \left|\tilde{\mathbf{I}}_{\mathrm{cor}}(\sigma)\right|^{2} \\
= & \frac{1}{4} B^{2}(\sigma) \kappa^{\prime 2}(\sigma) \mu_{12}^{2}(\sigma) \\
& +\frac{1}{4} B^{2}(-\sigma) \kappa^{\prime 2}(-\sigma) \mu_{12}^{2}(-\sigma) .
\end{aligned}
$$

It is clear, however, that Eq. (42) remains an idealization because it does not take into account the piston which perturbs the Fourier relationship. Thus we should not hope that it holds for each $\sigma$; however, provided that the piston is not too strong, we can assume that it only redistributes the fringe energy in the power spectrum, so that the relationship (42) is valid in an integral form:

$$
\begin{aligned}
S & =\int_{0}^{+\infty} \tilde{I}_{\text {cor }}^{2}(\sigma) \mathrm{d} \sigma \\
& =\int_{0}^{+\infty} \frac{1}{4} B^{2}(\sigma) \kappa^{\prime 2}(\sigma) \mu_{12}^{2}(\sigma) \mathrm{d} \sigma .
\end{aligned}
$$




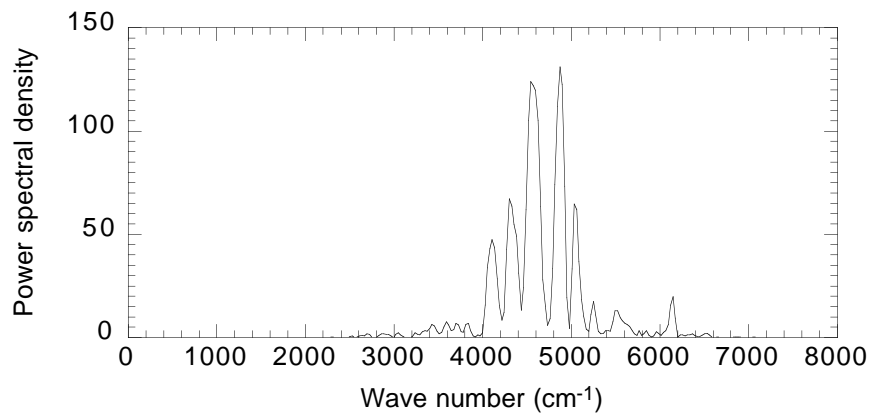

Fig. 6. Spectral power density of the corrected interferogram

Any departure from this approximation will be referred to as "piston noise", and we will see in Sect. 7.3 how its relative importance as a noise source can be evaluated.

Now if $\overline{\mu_{12}^{2}}$ is the integrated value, weighted by $B^{2}(\sigma) \kappa^{\prime 2}(\sigma)$, of the squared modulus of the coherence factor:

$\overline{\mu_{12}^{2}}=\frac{\int_{0}^{+\infty} B^{2}(\sigma) \kappa^{\prime 2}(\sigma) \mu_{12}^{2}(\sigma) \mathrm{d} \sigma}{\int_{0}^{+\infty} B^{2}(\sigma) \kappa^{\prime 2}(\sigma) \mathrm{d} \sigma}$

then $\overline{\mu_{12}^{2}}$ can be deduced from $S$ thanks to the relationship

$\overline{\mu_{12}^{2}}=\frac{4 S}{\int_{0}^{+\infty} B^{2}(\sigma) \kappa^{\prime 2}(\sigma) \mathrm{d} \sigma}$.

\subsubsection{The shape factor}

From Eq. (45) it appears that the value of $\overline{\mu_{12}^{2}}$ depends on a weighting factor

$F=\int_{0}^{+\infty} B^{2}(\sigma) \kappa^{\prime 2}(\sigma) \mathrm{d} \sigma$

which is intrinsic to the photometric behavior of the system and to the spectral intensity distribution of the source.

In practice, the quantity of interest is not the coherence factor per se but the visibility $V_{12}^{(\star)}$ of the source, which is obtained after the interferometer has been calibrated on a reference whose visibility $V_{12}^{\text {(ref) }}$ is well known. From the relationships (28) and (29), the modulus of the object visibility is given by:

$V_{12}^{(\star)}=V_{12}^{(\mathrm{ref})} \frac{\mu_{12}}{\mu_{12}^{(\mathrm{ref})}}$,

and the weighting factor disappears in the calibration process if the source and the reference have the same spectral intensity distribution ${ }^{2}$. Thus, provided the reference is adequately chosen, the determination of $F$ is not critical.

2 Rigorously speaking, this statement holds only as long as the chromaticism $\rho_{\sigma}$ of the injection efficiency, which appears implicitly in $F$ via the coefficient $\kappa^{\prime}(\sigma)$, does not vary signif-
Table 1. Shape factor computed for different sources, observed with a standard $K$ filter. For the stellar types, the calculation was based on infrared FTS spectra (Lançon \& Rocca-Volmerange 1992)

\begin{tabular}{ll}
\hline Source & $F_{B}(\mathrm{~cm})$ \\
\hline \hline & \\
\hline Stellar type G5III & $8.9510^{-4}$ \\
Stellar type M0III & $9.0810^{-4}$ \\
Stellar type M2V & $8.9810^{-4}$ \\
Stellar type M4III & $9.2010^{-4}$ \\
Stellar type M7V & $9.1710^{-4}$ \\
Stellar type M8III & $1.0210^{-3}$ \\
Blackbody $T=3000 \mathrm{~K}$ & $8.9110^{-4}$ \\
Blackbody $T=6000 \mathrm{~K}$ & $8.9410^{-4}$ \\
Blackbody $T=10000 \mathrm{~K}$ & $8.9610^{-4}$ \\
&
\end{tabular}

Usually the coefficient $F$ cannot be established directly because the quantity $\kappa^{\prime}(\sigma)$, defined by Eq. (32):

$\kappa^{\prime}(\sigma)=\kappa(\sigma) \frac{\sqrt{\eta_{P 1}(\sigma) \eta_{P 2}(\sigma)}}{\sqrt{\bar{\eta}_{P 1} \bar{\eta}_{P 2}}}$,

is difficult to measure at all wave numbers. We can make the reasonable approximation that $\kappa^{\prime}$ is achromatic and for all wave numbers

$\kappa^{\prime}(\sigma)=\bar{\kappa}=\sqrt{\bar{\kappa}_{1} \bar{\kappa}_{2}}$.

This assumption holds all the better as the couplers are less chromatic, but it is never rigorously true because of the chromaticism $\rho_{\sigma}$ of the injection efficiency that is included in the $\eta_{P j}$ coefficients (the global efficiency of the photometric channels). Nevertheless, any departure from the approximation can be seen, in the first order, as a purely instrumental effect, which is then included in the transfer function $T_{\mathrm{i}}$.

Once $\kappa^{\prime}(\sigma)$ has been set to a constant value the determination of $F$ is equivalent to the determination of another factor, $F_{B}$, which involves the source only and is defined by

$F_{B}=\frac{F}{\bar{\kappa}^{2}}$.

It will be called the shape factor because it depends on the shape of the normalized spectral intensity distribution:

$F_{B}=\int_{0}^{+\infty} B^{2}(\sigma) \mathrm{d} \sigma$.

icantly between the observations of the object and the reference. It might be possible that this effect becomes apparent with very large telescopes, because of differential refraction, if the two sources are observed at different zenithal distances (Coudé du Foresto 1994). 
Some numerical values of $F_{B}$ are given in Table 1 for different types of sources observed with a standard $K$ filter. The deeper the spectral features of the source, the larger the value of $F_{B}$. But if we except the very late type class M8III, the relative variation of the shape factor with the stellar spectral class is contained within $3 \%$. For a blackbody, $F_{B}$ depends very little on the temperature.

\section{Dealing with noisy data}

We consider here that the photometers in the fiber interferometer are detector noise limited. Each signal measurement is then affected by an additive, stationary noise, uncorrelated with the data. For each interferogram, a realization of the four noise signals is recorded in the background current sequences. These noise signals have to be taken into account when estimating the photometric signals and the squared coherence factor.

\subsection{Estimating the photometric signals}

The measurement $M_{P_{j}}$ of the signal produced by photometric detector $P_{j}$ is the sum of $P_{\mathrm{j}}(x)$ and the additive noise $b_{P_{j}}(x)$ :

$M_{P_{j}}(x)=P_{j}(x)+b_{P_{j}}(x)$.

The estimator $\widehat{P_{j}}$ of $P_{j}$ that minimizes the mean quadratic error with the actual signal is obtained by optimal filtering (Press et al. 1988):

$\widehat{P_{j}}(x)=\mathcal{F}^{-1}\left\{W_{j}(\sigma) \tilde{\mathbf{M}}_{P_{j}}(\sigma)\right\}$,

where $W_{j}(\sigma)$ is the Wiener filter, whose expression is, when the signal and the noise are uncorrelated:

$$
\begin{aligned}
W_{j}(\sigma) & =\frac{\left|\tilde{\mathbf{P}}_{j}(\sigma)\right|^{2}}{\left|\tilde{\mathbf{P}}_{j}(\sigma)\right|^{2}+\left|\tilde{\mathbf{b}}_{P_{j}}(\sigma)\right|^{2}} \\
& =\frac{\left|\tilde{\mathbf{M}}_{P_{j}}(\sigma)\right|^{2}-\left|\tilde{\mathbf{b}}_{P_{j}}(\sigma)\right|^{2}}{\left|\tilde{\mathbf{M}}_{P_{j}}(\sigma)\right|^{2}} .
\end{aligned}
$$

The power spectrum of the background current can be used to estimate $\left|\tilde{\mathbf{b}}_{P_{j}}(\sigma)\right|^{2}=\tilde{b}_{P_{j}}^{2}(\sigma)$.

The quality of the deconvolution signal $\widehat{P}=\sqrt{\widehat{P_{1}} \widehat{P_{2}}}$ is critical to achieve a good interferogram correction. What matters is not the average of $\widehat{P}(x)$ but rather its smallest value: if at the minimum the local signal to noise ratio is too low, then the division is numerically unstable and the interferogram correction process is useless. Therefore it is necessary to adopt a selection scheme: if the minimum value of $\widehat{P_{1}}$ or $\widehat{P_{2}}$ is below a certain rejection threshold, then the interferogram is discarded and does not lead to a fringe visibility measurement. For a photometric signal $\widehat{P_{j}}$, the rejection threshold can be expressed as a multiple of the standard deviation $\sigma_{b_{j}}$ of the corresponding background sequence, filtered by $W_{j}$.

There is a trade-off in the choice of the rejection threshold, between rejecting only a few interferograms and having a better correction. For sufficiently good data this choice is not critical, as the correction quality does not significantly depend on the minimum value of the $\widehat{P}_{j}$ as long as this value is greater than $10 \sigma_{b_{j}}$. For faint objects or in bad seeing conditions, one may have to use rejection thresholds as low as $3 \sigma_{b_{j}}$.

\subsection{Estimating the squared coherence factor}

We should now seek an estimator of the coherence factor that is not biased by detector noise. The signal $M(x)$ actually measured at the output of the interferometric detector contains an additive noise $b_{\mathrm{I}}(x)$ :

$M(x)=I(x)+b_{\mathrm{I}}(x)$.

If the data processing described in the preceding sections is applied to $M$, it leads in the wave number space to

$\tilde{\mathbf{M}}_{\text {cor }}(\sigma)=\tilde{\mathbf{I}}_{\text {cor }}(\sigma)+\tilde{\mathbf{b}}_{\text {cor }}(\sigma)$,

and, since the signal and the noise are uncorrelated, their moduli add quadratically:

$\tilde{M}_{\text {cor }}^{2}(\sigma)=\tilde{I}_{\text {cor }}^{2}(\sigma)+\tilde{b}_{\text {cor }}^{2}(\sigma)$,

which yields, in the integral form that is assumed to be valid when there is differential piston:

$\int_{0}^{+\infty} \tilde{M}_{\text {cor }}^{2}(\sigma) \mathrm{d} \sigma=\int_{0}^{+\infty} \tilde{I}_{\text {cor }}^{2}(\sigma) \mathrm{d} \sigma+\int_{0}^{+\infty} \tilde{b}_{\text {cor }}^{2}(\sigma) \mathrm{d} \sigma(58)$

$S_{M}=S+S_{b}$

An estimator $\widehat{S_{b}}$ of the noise integral is obtained by applying to the current signal the same treatment that was used to process $M$. A better estimator is obtained by processing many realizations of the interferometric background current (for example, all the background sequences of a complete batch), and averaging the resulting power spectra. Eq. (58) then provides an estimator of $S$ :

$\widehat{S}=S_{M}-\widehat{S_{b}}$,

from which Eq. (45) enables us to establish an unbiased estimator of the squared modulus of the coherence factor, averaged over the optical bandpass:

$\widehat{\mu_{12}^{2}}=\frac{4 \widehat{S}}{\bar{\kappa}^{2} F_{B}}$.

The quantity $\widehat{\mu_{12}^{2}}$ is the final result of the data reduction process on a single interferogram. 


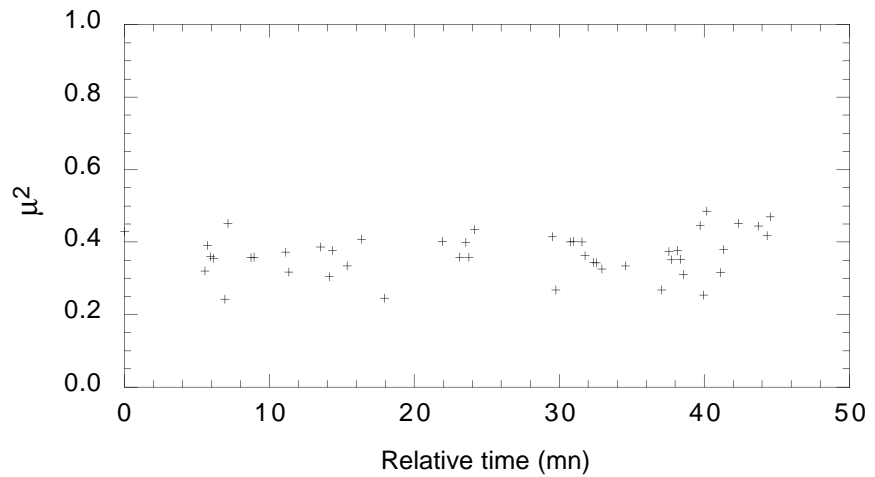

Fig. 7. Result of the data reduction on a batch of 122 interferograms of $\alpha$ Boo. The adopted shape factor was $F_{B}=910^{-4} \mathrm{~cm}$. Because of mediocre seeing conditions, the rejection threshold was set to 3 : only 47 interferograms met this level and led to a measurement of the squared coherence factor. For that batch the final estimate of the squared coherence factor is $\mu^{2}=0.367 \pm 0.0084$

\subsection{Statistical error and noise sources}

A batch of interferograms leads to a collection of $n$ measurements of the fringe visibility. An example is shown in Fig. 7. The final estimate of the squared coherence factor is the average of the $\widehat{\mu_{12}^{2}}$, and if $\sigma_{\mu^{2}}$ is the standard deviation of the estimator of $\overline{\mu_{12}^{2}}$, then the standard error is

$\epsilon_{\mu^{2}}=\frac{\sigma_{\mu^{2}}}{\sqrt{n}}$

The final error estimate for $\widehat{\mu_{12}^{2}}$ should include the uncertainties on $\bar{\kappa}$ and $F_{B}$.

Fluctuations in the measurement of $\overline{\mu_{12}^{2}}$ can be attributed to three main noise sources:

- Detector noise: because of statistical fluctuations of the noise in the interferometric detector, the estimation $\widehat{S_{b}}$ is not exactly the noise integral $S_{b}$;

- Deconvolution noise: because of residual noise in the optimally filtered photometric signals, the interferometric signal is divided by an estimation $\widehat{P}$ that differs from the true deconvolution signal $P$. An adequate rejection threshold should ensure that the deconvolution noise is low (or even negligible);

- Piston noise: in the presence of differential piston, the integral relationship (43) is only an approximation.

Another source of "noise" results from the incomplete realization of the chromaticism assumption developed in Sect. 3.2. It will not be discussed here, since in the standard operating conditions of an infrared fiber interferometer $\left(d / r_{0} \leq 4\right.$, bandpass limited by the atmospheric transmission window) its effects are always negligible.

Although we have so far considered only one interferometric output, each scan provides two measurements of $\overline{\mu_{12}^{2}}$. Coherence factor measurements on each output must be treated separately because each channel has its own instrumental transfer function. But the correlation between the two channels can tell us about the relative importance of the noise sources.

Detector noise is fully uncorrelated between the two interferometric outputs. Conversely, the differential piston perturbations are exactly the same on each output. Deconvolution noise is very strongly correlated since the deconvolution signal is the same for both channels, but not fully correlated because the deconvolution is applied to two separate measurements of the interferometric signal, each one affected by independent noise.

Let $M_{\mu^{2}}^{(1)}$ be the estimator of $\overline{\mu_{12}^{2}}$ for Channel 1 (Eq. 61) and $M_{\mu^{2}}^{(2)}$ for Channel 2. The following holds without making any assumption on the nature of the noise sources:

$\left\{\begin{array}{l}M_{\mu^{2}}^{(1)}=\overline{\mu_{12}^{2}}+b_{\mathrm{c}}+b_{\mathrm{uc}}^{(1)} \\ M_{\mu^{2}}^{(2)}=\overline{\mu_{12}^{2}}+b_{\mathrm{c}}+b_{\mathrm{uc}}^{(2)}\end{array}\right.$,

where $b_{\mathrm{c}}$ is the correlated part of the noise for Channel $i$, and $b_{\mathrm{uc}}^{(i)}$ is the uncorrelated part. For each interferogram, it is possible to measure the difference $M_{\Delta \mu^{2}}$ between the two estimators, i.e.

$$
\begin{aligned}
M_{\Delta \mu^{2}} & =M_{\mu^{2}}^{(2)}-M_{\mu^{2}}^{(1)} \\
& =b_{\mathrm{uc}}^{(2)}-b_{\mathrm{uc}}^{(1)}
\end{aligned}
$$

and for a batch of scans the variance of $M_{\Delta \mu^{2}}$ is the sum of the variances of the uncorrelated noises:

$\sigma_{\Delta \mu^{2}}^{2}=\sigma_{\mathrm{uc} 1}^{2}+\sigma_{\mathrm{uc} 2}^{2}$

If two photometers of the same type have been employed to measure the interferometric outputs, the statistics of the uncorrelated noise is the same on both channels. It is then possible to determine the variance of the uncorrelated noise:

$\sigma_{\mathrm{uc}}^{2}=\frac{\sigma_{\Delta \mu^{2}}^{2}}{2}$.

The variance of the correlated noise follows immediately from Eq. (63):

$\sigma_{\mathrm{c}}^{2}=\sigma_{\mu^{2}}^{2}-\sigma_{\mathrm{uc}}^{2}$

From what was said above, we can with a good approximation identify $\sigma_{\mathrm{uc}}^{2}$ with the detector noise, and $\sigma_{\mathrm{c}}^{2}$ with the joint contribution of piston and deconvolution noise. Thus simultaneous measurements of both interferometric outputs make it possible to tell the relative contribution of detector noise to the total noise.

This is useful to work out the optimum fringe speed $v$, which is the result of a compromise. On the one hand detector noise increases with $v$, because for a given optical bandpass the fringe signal is spread over a wider frequency bandpass. On the other hand the faster the interferogram 
is scanned, the more frozen is the seeing for the duration of the scan, and the piston perturbations are smaller. In the $\alpha$ Boo example, $44 \%$ of the noise variance is uncorrelated, which means that no single source dominates the noise.

\section{Practical considerations}

\subsection{Signal synchronization}

Before beginning the data reduction process it is important to ensure that all recorded signals are synchronized with an accuracy better than one sample interval $\delta x$. This might not be true if for example the interferometric and photometric signals are delayed by different electronics (e.g., analog filters) before being digitized and recorded. In that case, the amount of desynchronization can be evaluated by looking at the position of the correlation peak between $I$ and the best linear fit to $I$ of $P_{1}$ and $P_{2}$. A corresponding offset is then applied to the relevant signals; this leaves a few samples at the beginning or the end of the sequence as undefined.

\subsection{Apodization}

An apodization of the corrected interferometric signal is necessary for several reasons: it solves the problem of undefined samples for the signals that have been synchronized, and it removes potential boundary effects when Fast Fourier Transforms (FFTs) are performed. Because it reduces the effective length of the sequence, apodization also contributes to attenuate the detector and piston noises.

Therefore before measuring $\widehat{S}$, the signal $M_{\text {cor }}(x)$ is multiplied by an apodization window $A(x)$. Any smoothly varying function could be employed; the following function was chosen (assuming the OPD scan spans the interval $[-\Delta x / 2, \Delta x / 2])$ :

$A(x)=\left\{\begin{array}{ll}0 & |x|>\left|x_{2}\right| \\ \cos \left[\frac{\pi}{4}\left(\frac{|x|-\left|x_{1}\right|}{\left|x_{2}-x_{1}\right|}\right)\right] & \left|x_{1}\right| \leq|x| \leq\left|x_{2}\right| . \\ 1 & |x|<\left|x_{1}\right|\end{array}\right.$.

Thus the window blocks out the signal for $|x|>\left|x_{2}\right|$, and provides a smooth transition to full transmission for $\left|x_{1}\right| \leq$ $|x| \leq\left|x_{2}\right|$. The choice of $x_{1}$ and $x_{2}$ is not critical and depends essentially on the number of fringes that can be seen above the noise level of the interferometric detector. In the $\alpha$ Boo example, the signal is blocked out for $0.2 \Delta x$ on each side of the scans, and the length of the transition regions is $0.1 \Delta x$.

\subsection{Computing the Wiener filters}

For each photometric signal, the corresponding optimal filter is estimated by using the power spectrum of the background current sequence as an estimator $\widehat{\tilde{b}_{P_{j}}^{2}}(\sigma)$ of the noise power spectral density. It is expected that the value of $\widehat{W}$ is close to 1 for low frequencies where the photometric signal is strong, and decreasing to zero at higher frequencies where detector noise dominates.

A problem arises at some higher frequencies when, because of statistical fluctuations in the noise power density, the estimated noise power $\widehat{\mathrm{b}_{P_{j}}^{2}}(\sigma)$ is smaller than the measured signal power $\tilde{M}_{P_{i}}^{2}(\sigma)$. This would imply a negative value for the Wiener filter. To avoid this, the estimated Wiener filter is defined as such:

$\widehat{W}(\sigma)=\left\{\begin{array}{ll}1 & \sigma=0 \\ \frac{\tilde{M}_{P_{j}}^{2}(\sigma)-\widehat{\hat{b}_{P_{j}}^{2}}(\sigma)}{\tilde{M}_{P_{j}}^{2}(\sigma)} & \sigma \leq \sigma_{0} \\ 0 & \sigma>\sigma_{0}\end{array}\right.$,

where $\sigma_{0}$ is the smallest wave number that meets the condition $\tilde{M}_{P_{j}}^{2}(\sigma)<\widetilde{b}_{P_{j}}^{2}(\sigma)$. The value of $\widehat{W}(\sigma)$ is forced to 1 at the continuum to maintain the average value of the photometric signal.
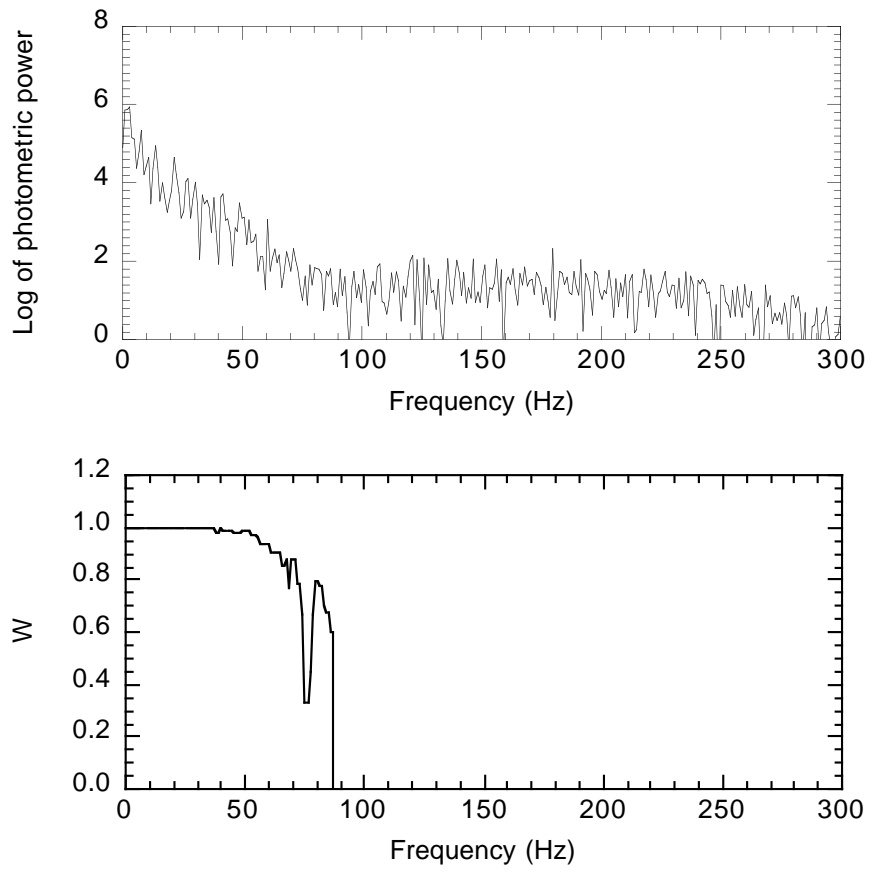

Fig. 8. Spectral power of a photometric signal (top, note the logarithmic scale) and its associated Wiener filter (bottom)

Figure 8 shows an example of photometric power density and the associated $\widehat{W}$.

\subsection{Numerical evaluation of $\widehat{S}$}

Here is a link from the continuous world of functions to the discrete world of computer data. The physical signal $M(x)$ is sampled every $\delta x$ and recorded in the computer as a series of numbers $y_{i}(i=0,1, \ldots, N-1)$ such that:

$y_{i}=M(i \delta x)$. 
Data reduction is performed by the computer on the $y_{i}$ series. The continuous Fourier transform is replaced by a Fast Fourier Transform, which produces a new series of $N$ numbers (harmonics) $\mathbf{Y}_{k}$. Positive frequencies are represented by harmonics $\mathbf{Y}_{1}$ to $\mathbf{Y}_{N / 2}$ (with $\mathbf{Y}_{N / 2}$ being the Nyquist harmonic). If the physical signal was correctly sampled (i.e. if $\tilde{M}(\sigma)=0$ for $\sigma \geq N \delta \sigma / 2$ ), then each $\mathbf{Y}_{k}$ $(k=0, \ldots, N / 2)$ is linked to $\tilde{\mathbf{M}}(\sigma)$ by the relationship (Brigham 1974):

$\mathbf{Y}_{k}=N \delta \sigma \tilde{\mathbf{M}}(k \delta \sigma)$.

Therefore the numerical data reduction process yields a final series $\mathbf{Z}_{k}$ of complex numbers whose moduli for $k=$ $0, \ldots, N / 2$ are linked to $\tilde{\mathbf{M}}_{\text {cor }}(\sigma)$ by:

$Z_{k}^{2}=N^{2} \delta \sigma^{2} \tilde{M}_{\text {cor }}^{2}(k \delta \sigma)$.

The integral $S_{M}$ of the squared modulus of $\tilde{\mathbf{M}}_{\text {cor }}$ can be evaluated numerically using the trapezoidal rule:

$$
\begin{aligned}
S_{M} & =\int_{0}^{+\infty} \tilde{M}_{\mathrm{cor}}^{2}(\sigma) \mathrm{d} \sigma \\
& \simeq \frac{\delta \sigma}{2}\left(\tilde{M}_{\mathrm{cor}}^{2}(0)+\tilde{M}_{\mathrm{cor}}^{2}\left(\frac{N}{2} \delta \sigma\right)\right)+\sum_{k=1}^{\frac{N}{2}-1} \tilde{M}_{\mathrm{cor}}^{2}(k \delta \sigma) \delta \sigma
\end{aligned}
$$

It follows that the numerical value of $S_{M}$ can be computed from the $Z_{k}$ series with:

$S_{M}=\frac{1}{N^{2} \delta \sigma}\left[\frac{1}{2}\left(Z_{0}^{2}+Z_{N / 2}^{2}\right)+\sum_{k=1}^{\frac{N}{2}-1} Z_{k}^{2}\right]$.

The numerical evaluation of $\widehat{S_{b}}$ is performed in a similar way.

\subsubsection{Choice of integration range}

In principle, to minimize the statistical fluctuations of $S_{b}$ the integration boundaries could be reduced to the wave number range corresponding to the optical bandpass of the system. In practice, a wider range is required because the piston perturbations spread the interferometric signal over a range larger than the nominal bandpass. A compromise has to be adopted, between not risking to miss part of the signal spread by the piston, and reducing the statistical fluctuations of $S_{b}$. Experience proved that this choice is not critical. In the $\alpha$ Boo example, integration was performed between 3000 and $6000 \mathrm{~cm}^{-1}$, to be compared with an optical bandpass in the $K$ band of $4000-5000 \mathrm{~cm}^{-1}$.

\section{Generalization}

Much of the data reduction procedure detailed in this paper can be applied even if the photometric signals $P_{1}$ and $P_{2}$ cannot be measured (if for example the fiber unit contains only a single $X$ coupler), provided that the scintillation noise and the fringe signal do not overlap in the

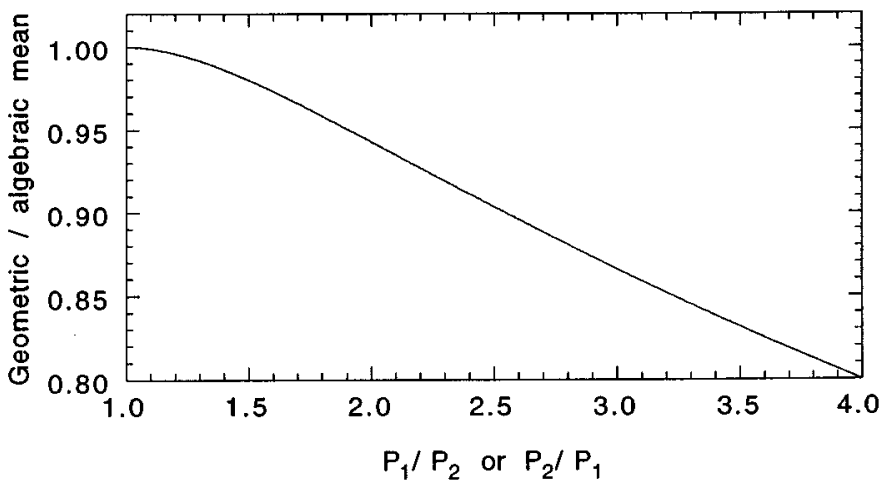

Fig. 9. Error introduced in the pseudo correction when the geometric mean of $P_{1}$ and $P_{2}$ is replaced by the arithmetic mean

spectrum of $I(x)$. In that case Sect. 4 is no longer relevant and a simple filtering can separate the high frequency fringe signal $I_{\mathrm{HF}}(x)$ from the low frequency scintillation noise $I_{\mathrm{BF}}(x)=\bar{\kappa}_{1} P_{1}(x)+\bar{\kappa}_{2} P_{2}(x)$. We can then build a "pseudo corrected" interferometric signal $\tilde{\mathbf{I}}_{\text {cor }}^{\prime}(\sigma)$ as:

$$
\begin{aligned}
\tilde{\mathbf{I}}_{\text {cor }}^{\prime}(\sigma)= & \frac{1}{2} \mathcal{F}\left\{\frac{I_{\mathrm{HF}}(x)}{0.5 I_{\mathrm{BF}}(x)}\right\} \\
\simeq & \frac{1}{2} B(\sigma) \mu_{12}(\sigma) \mathrm{e}^{j \Phi_{12}(\sigma)} \\
& +\frac{1}{2} B(-\sigma) \mu_{12}(-\sigma) \mathrm{e}^{-j \Phi_{12}(-\sigma)}
\end{aligned}
$$

The pseudo correction consists in approximating the geometric mean of $\bar{\kappa}_{1} P_{1}$ and $\bar{\kappa}_{2} P_{2}$ by the arithmetic mean. The ratio of the geometric mean to the arithmetic mean is plotted in Fig. 9. The approximation leads to a systematic underestimate of the coherence factor, the amount of which depends on the statistics of the scintillation.

By extension, the same procedure can be used for any pupil plane interferometer, provided that the data be obtained by recording a scan around the zero OPD. It represents then an approach complementary to what was proposed by Benson et al. (1995). In a dioptric interferometer (using mirrors and beamsplitters) the photometric signals, affected only by atmospheric scintillation, are equal in average (assuming identical pupils) and much more stable than in a fiber interferometer. Then the bias introduced by the approximation in Eq. (75) is usually negligible. Indeed the error is less than $1 \%$ if $P_{1}$ and $P_{2}$ do not differ by more than $30 \%$. With a dioptric interferometer however, an atmospheric transfer function $T_{\mathrm{a}}$ is involved between the measured coherence factor and the object visibility (Eq. 1), and the classical calibration problems remain.

\section{Conclusion}

Single-mode fibers transform phase distortions into intensity fluctuations in a stellar interferometer. This is an worthy trade-off, as the scintillation can easily be monitored, 
and we have seen how these signals are used to correct interferograms from the photometric fluctuations. The correction is essentially a deconvolution, and a criterion was established to reject those scans for which this operation is not numerically stable. Corrected interferograms then lead to coherence factor measurements that are linked to the object visibility by a purely instrumental transfer function, where atmospheric turbulence is not involved. This feature greatly improves the accuracy of object visibility measurements.

One turbulence mode, however, is not filtered out by the fibers: the differential piston, which can be either removed by a fringe tracking system or reduced (at the expense of increasing detection noise) by scanning the OPD more rapidly. If there is no piston we saw that the data contain both spatial and spectral information on the source. This is the basis of double Fourier interferometry, a very promising technique for instruments which are equipped with a fringe tracker. Double Fourier interferometry with fibers can be achieved only if time and wavelength are effectively independent variables in the starlight injection function, and further work remains to be done in order to determine what this implies in terms of optical bandpass and input wavefront quality.

When piston perturbations exist, the phase and spectral information on the source is lost: then only the modulus of the coherence factor, integrated over the optical bandpass, can be accessed, through the amount of energy in the high frequency part (fringe signal) of the Fourier transform of the interferogram. An expression was derived for a noise bias free estimator of the squared modulus of the coherence factor. It depends (in a non critical way) of a spectral weighting factor, whose numerical value is given for different types of sources. The dispersion of the measured squared coherence factor is caused mainly by detector and piston noise, in relative proportions that can be evaluated.

The end result is an object visibility measurement with a statistical accuracy than can be better than $1 \%$ in a few tens of interferograms. The large gain in precision provided by single-mode fibers over conventional (dioptric) optics opens long baseline interferometry to a whole new class of astrophysical problems (Perrin et al. 1977).

Acknowledgements. Most of this work was performed as the author was a Ph.D. student at the Département de Recherches Spatiales (DESPA) of Observatoire de Paris.

\section{References}

Benson J., Dyck H., Howell R., 1995, Appl. Opt. 34, 51

Born M., Wolf E., 1980, Principle of Optics. Pergamon Press, Oxford

Brigham E.O., 1974, The Fast Fourier Transform. PrenticeHall, Englewood Cliffs, NJ

Carleton N.P., Traub W.A., Lacasse M.G., et al., 1994, in: Astronomical Telescopes and Instrumentation for the 21st century, SPIE 2200, Kona, Hawaii, p. 152

Claret A., Mariotti J.-M., Léna P., 1991, in: Beckers J., Merkle F. (eds.) High-resolution imaging by interferometry II. ESO, Garching, p. 835

Coudé du Foresto V., 1994, Interférométrie astronomique infrarouge par optique guidée monomode. $\mathrm{PhD}$ thesis, Université Denis Diderot (Paris 7)

Coudé du Foresto V., Perrin G., Boccas M., 1995, A\&A 293, 278

Coudé du Foresto V., Ridgway S., 1991, in: Beckers J., Merkle F. (eds.) High-resolution imaging by interferometry II. ESO, Garching, p. 731

Fried D., 1966, JOSA 56, 1372

Goodman J.W., 1985, Statistical Optics. John Wiley \& Sons, New York

Itoh K., Ohtsuka Y., 1986, JOSA 3, 94

Lançon A., Rocca-Volmerange B., 1992, A\&AS 96, 593

Mariotti J.-M., 1993. In: Alloin D., Mariotti J.-M. (eds.) Adaptive Optics for Astronomy. Kluwer Academic Publishers, Cargèse, Corsica, p. 309

Mariotti J.-M., Ridgway S.T., 1988, A\&A 195, 350

Neumann E.-G., 1988, Single-Mode Fibers. Springer-Verlag, Berlin

Noll R.J., 1976, JOSA 66, 207

Perley R.A., Schwab F.R., Bridle A.H., 1985, Synthesis Imaging: Course Notes from an NRAO Summer School. NRAO, Socorro, New Mexico

Perrin G., 1997, A\&A (in preparation)

Perrin G., Coudé du Foresto V., Benson J., et al., 1995, in: Barden S.C. (ed.) Fiber Optics in Astronomical Applications. SPIE, Orlando, p. 120

Perrin G., Coudé du Foresto V., Ridgway S., et al., 1997 (submitted to A\&A)

Press W.H., Flannery B.P., Teukolsky S.A., Vetterling W.T., 1988, Numerical Recipes in C. Cambridge University Press, Cambridge

Rohloff R.-R., Leinert C., 1991, in: Beckers J., Merkle F. (eds.) High-resolution imaging by interferometry II. ESO, Garching, p. 1303

Shaklan S., Roddier F., 1988, Appl. Opt. 27, 2334

Shao M., Colavita M.M., Hines B.E., et al., 1988, A\&A 193, 357 